\title{
Características esqueletales para la determinación cualitativa del biotipo facial en telerradiografía lateral: Estudio Piloto.
}

\section{Skeletal characteristics for subjective determination of facial type in cephalometric $x$-ray: a pilot study.}

\author{
Rolando Schulz-Rosales ${ }^{1 *}$, Macarena Núñez-Guerrero², Bárbara Cerda-Peralta ${ }^{3}$, Macarena Rivera-Rothgaenger ${ }^{3}$, \\ Enrique Ponce de León ${ }^{3}$, Jimena López-Garrido², Stefan Danilla-Enei", Fernando Romo-Ormazábal' ${ }^{1}$.
}

1. Clínica Integral del Adulto, Dpto. Prótesis, Facultad de Odontología, Universidad de Chile. 2. Práctica privada, Santiago, Chile.

3. Clínica Integral del Adulto, Dirección Escuela de Pregrado, Facultad de Odontología, Universidad de Chile.

4. Departamento de Cirugía, Facultad de Medicina, Universidad de Chile.

* Correspondencia autor. Dr. Rolando Schulz Rosales, Profesor Asistente | Facultad de odontología Universidad de Chile | E-mail: rschulz@odontologia.uchile.cl | Teléfono: +562 29781715 | Dirección postal: Sergio Livingstone Pohlhammer $N^{\circ} 943$ (ex Olivos)

Trabajo recibido el 22/08/2016.

Aprobado para su publicación el 29/12/2016

\section{RESUMEN}

Objetivo: Determinar parámetros visuales sencillos para la evaluación de características esqueletales relacionadas con el biotipo facial en una telerradiografía lateral. Material y método: Estudio transversal descriptivo. Se confeccionó una guía visual impresa acerca de ocho características morfológicas del esqueleto facial visibles en la telerradiografía de perfil. Ésta fue entregada a un grupo de 69 estudiantes de Odontología de $5^{\circ}$ año sin estudios previos en cefalometría, quienes la analizaron durante 10 minutos. Inmediatamente se les mostraron una serie de telerradiografías, y se les pidió que evaluaran las 8 características a través de una serie de opciones presentadas en una escala visual que se correspondía con la guía estudiada. Las respuestas fueron registradas utilizando un sistema digital de cliqueras. Los datos fueron analizados con el método Rasch para determinar la dificultad de los ítems, estableciéndose el grado de dificultad a través del Logit. Resultados: Los estudiantes lograron identificar y graduar sin dificultad 5 de las 8 características presentadas. Éstas fueron: Proyección del mentón $(-2,49)$, Divergencia facial $(-2,85)$, Ancho de la rama mandibular $(-1,97)$, Escotadura sigmoidea $(-0,96)$ y Relación de la rama con el cuerpo mandibular $(-0,06)$. Conclusión: Se determinó que hay 5 características de la morfología ósea facial relacionadas con el biotipo que se pueden identificar y graduar sin dificultad por estudiantes de pregrado de odontología. Ésta información podría ser utilizada para construir una escala sencilla de identificación del biotipo facial.

PALABRAS CLAVE:

Cefalometría; Crecimiento y desarrollo; Cara; Mandíbula; Cefalometría lateral.

Rev. Clin. Periodoncia Implantol. Rehabil. Oral Vol. 11(3); 164-166, 2018.

\section{ABSTRACT}

Aim: Determine simple visual parameters to evaluate skeletal characteristics related to facial types in lateral teleradiographs. Materials and Methods: Descriptive crosssectional study. A printed visual guideline about the eight visible morphological characteristics of the facial skeleton in cephalometric $\mathrm{x}$-rays was created. The guideline was given to 69 undergraduate dental students (fifth year) without previous studies in cephalometric to analyze it for 10 minutes. Immediately after, they were shown a series of teleradiographs and were asked to evaluate 8 characteristics through a series of options presented in a visual scale according to the guideline. The answers were recorded through a digital audience response system (clickers). The data obtained were analyzed with the Rasch method to assess each item level of difficulty, establishing the Logit number for each one. Results: The students identified and graded without difficulty 5 of the 8 characteristics. These were: Chin projection (-2.49), Facial divergence $(-2.85)$, Mandibular ramus width $(-1.97)$, Sigmoid notch $(-0.96)$ and Mandibular body with ramus relationship $(-0.06)$. Conclusions: Five facial osseous morphological characteristics related to the facial types can be identified and graded without difficulty by undergraduate dental students. This information may be used to create a facial type identification scale.

KEY WORDS:

Cephalometric analysis; Growth and Development; Face; Jaw; Orthodontic; Lateral cephalogram.

Rev. Clin. Periodoncia Implantol. Rehabil. Oral Vol. 11(3); 164-166, 2018. 


\section{INTRODUCCIÓN}

El biotipo facial corresponde a las características morfológicas y funcionales que determinan la dirección del crecimiento y comportamiento funcional del macizo cráneo-facial de un individuo, cuya expresión es hereditaria y modificada por el ambiente ${ }^{(1)}$. En definitiva, nos indica la predominancia entre las dimensiones verticales y horizontales en un rostro(2).

El biotipo facial es de gran utilidad en las diferentes áreas de la odontología. Es utilizado en la toma de decisiones terapéuticas por ejemplo, como parámetro para la selección dentaria en prótesis ${ }^{(3)}$ y para determinar el anclaje en ortodoncia ${ }^{(4)}$.

Existen variados métodos para la determinación del biotipo facial. Algunos basados en el examen clínico ${ }^{(1)}$, fotográfico ${ }^{(5)}$ y otros en el análisis de la telerradiografía lateral( ${ }^{(6)}$. Existen normas cefalométricas que permiten la determinación del biotipo facial. Entre ellas encontramos: el análisis VERT de Ricketts ${ }^{(1)}$, Cuociente de Björk-Jarabak ${ }^{(7)}$ y la divergencia de las bases óseas de Downs-Steiner $r^{(9)}$.

EI VERT de Ricketts corresponde a un coeficiente de variación ${ }^{(9)}$ que establece numéricamente el tipo y la cantidad de crecimiento vertical del esqueleto facial provocado por la rotación mandibular. Es un análisis multifactorial, donde son evaluados cinco parámetros, que representan 3 aspectos principales: la altura facial, la rotación y desarrollo mandibular ${ }^{(1)}$. Una desventaja del VERT es que se realiza con aplicaciones de software especializado. Es posible también realizarlo manualmente, pero requiere mucho tiempo y es difícil para aquellos profesionales con poca experiencia.

Por otro lado, Jarabak categorizó la morfología facial en tres patrones según el Cuociente de Jarabak o "Jarabak Ratio" (FHR), correspondiente a la proporción entre la altura facial posterior (S-Goc) y la altura facial anterior (N-Me). Esta relación representa el grado de divergencia del plano mandibular respecto al plano silla-Nasion, definiendo de ese modo tres patrones de crecimiento facial $^{(7)}$.

La divergencia de las bases óseas de Downs-Steiner corresponde al ángulo SN-Go-Gn, el cual expresa la convergencia o divergencia de la base craneal con la mandíbula ${ }^{(9)}$.

Jarabak y Fizzell describieron características esqueletales de los distintos biotipos faciales ${ }^{(7)}$. Sin embargo, si bien en su texto se realiza una descripción detallada de los patrones faciales, no especifica el nombre de cada biotipo como se conoce actualmente, sino que son clasificados como tipo I, II y III. La literatura actual permite identificar que cada uno de esos patrones son descripciones de lo que actualmente conocemos como mesofacial, braquifacial y dolicofacial(10). Estas características esqueletales descritas por los autores pueden ser apreciadas visualmente, sin la necesidad de realizar trazados cefalométricos. Por ejemplo, en el braquifacial el mentón se encuentra proyectado hacia delante y la escotadura maseterina se aprecia bien marcada.

Un método basado sólo en la apreciación cualitativa del esqueleto facial permitiría a un odontólogo no especialista con solo diez minutos de instrucción, visualizar parámetros que le permitan determinar el biotipo facial del paciente de manera sencilla en una telerradiografía de perfil, sólo con conocimientos básicos de anatomía y radiología.

\section{MATERIAL Y MÉTODO}

Se confeccionó una guía visual (Fig. 1) con 8 características faciales basadas en las observaciones de Jarabak y Fizzel|(7), debido a su facilidad para reconocerlas en la telerradiografía. Se entregó a 69 estudiantes de quinto año de la Facultad de Odontología de la Universidad de Chile sin formación previa en cefalometría. Los alumnos tuvieron diez minutos para estudiar el documento, luego se proyectaron dos telerradiografías donde se apreciaban los siguientes ítems a evaluar (Fig. 2):

1. Proporción entre el cuerpo y la rama mandibular.

2. Ángulo goníaco (abierto o cerrado).

3. Relación de altura entre cóndilo y coronoides (cóndilo más alto o cóndilo y coronoides de igual altura).

4. Escotadura antegonial (marcada o poco marcada).

5. Escotadura sigmoidea (muy profunda o poco profunda).

6. Prominencia del mentón (proyectado verticalmente o proyectado horizontalmente).

7. Ancho de la rama mandibular (ancha o angosta).

8. Divergencia entre la base craneal anterior (S-N) y el plano mandibular (GnGo) (divergentes o paralelos).

Los participantes asignaron un puntaje de 1 a 5 , donde 1 correspondía a una característica braquifacial marcada y 5 a una característica dolicofacial acentuada.

La calificación de algunos ítems fue invertida intencionalmente para evitar sesgos. A los participantes no se les informó acerca del objetivo del braquifacial

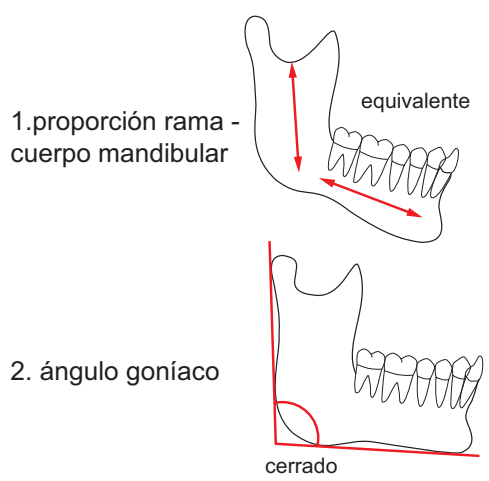

3.altura cóndilo coronoides antegonial

4.escotadura

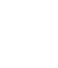

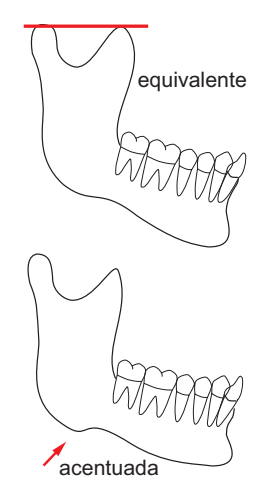

5.escotadura sigmoídea del mentón
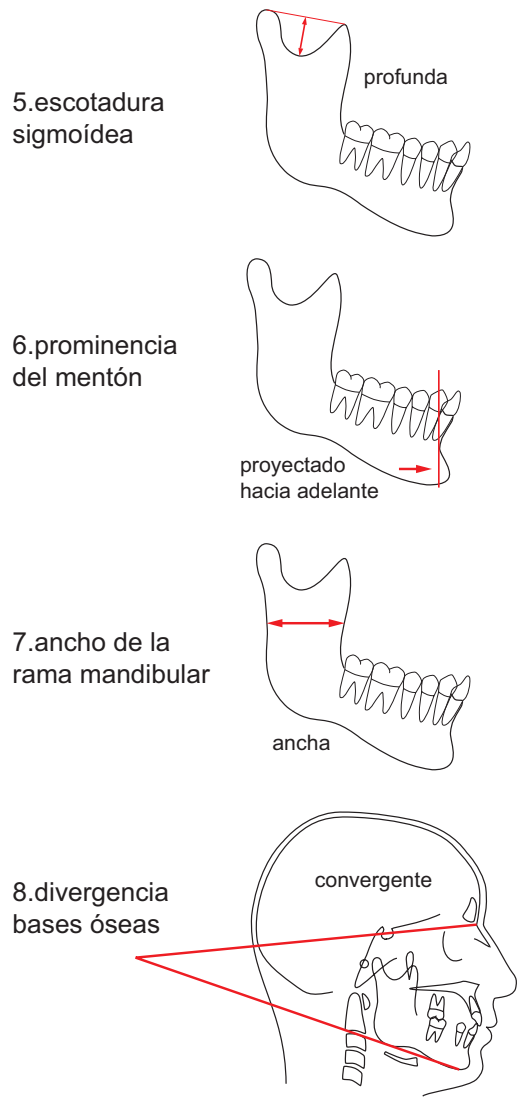

Figura 1. Características esqueletales de los diferentes biotipos

dólicofacial
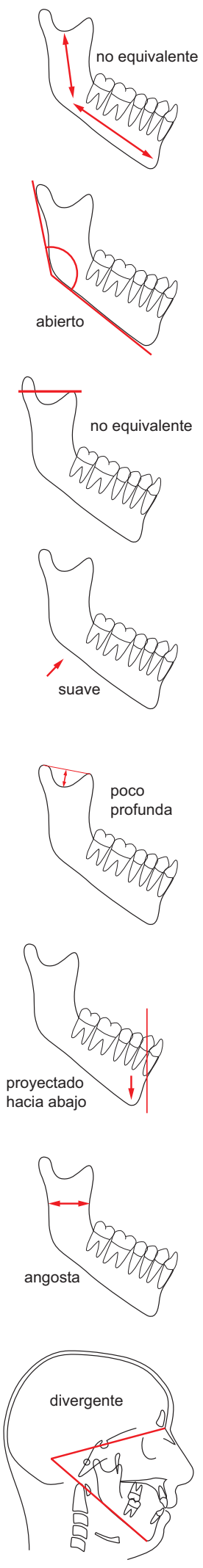


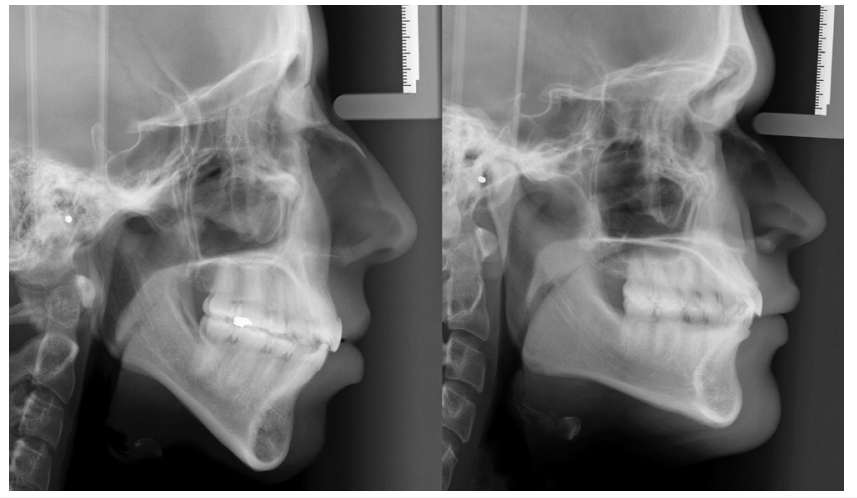

Figura 2. Telerradiografías de perfil de un individuo braquifacial (izquierda) y un dolicofacial (derecha).

estudio para no influenciar sus respuestas.

Los datos fueron recolectados a través del sistema de respuesta de audiencia (ARS) mediante cliqueras.

\section{RESULTADOS}

Se aplicó el análisis estadístico Rasch mediante el software JMetrik ${ }^{\mathrm{MR}}$ evaluándose la dificultad de cada ítem mediante el dígito "Logit"(8,11)

Al analizar la dificultad de los ítems (Gráfico 1), se observa que los ítems Divergencia facial, Proyección del mentón, y Ancho de la rama mandibular se clasifican con dificultad "fácil o muy fácil" (Logit < -1); los ítems Escotadura sigmoídea y Relación de la rama y cuerpo mandibular se clasifican con dificultad "adecuada" (Logit entre -1 y +1 ); los ítems Cóndilo/Coronoides, Ángulo goniaco y Escotadura antegonial se clasifican con dificultad "difícil o muy difícil" (Logit $>+1$ ).

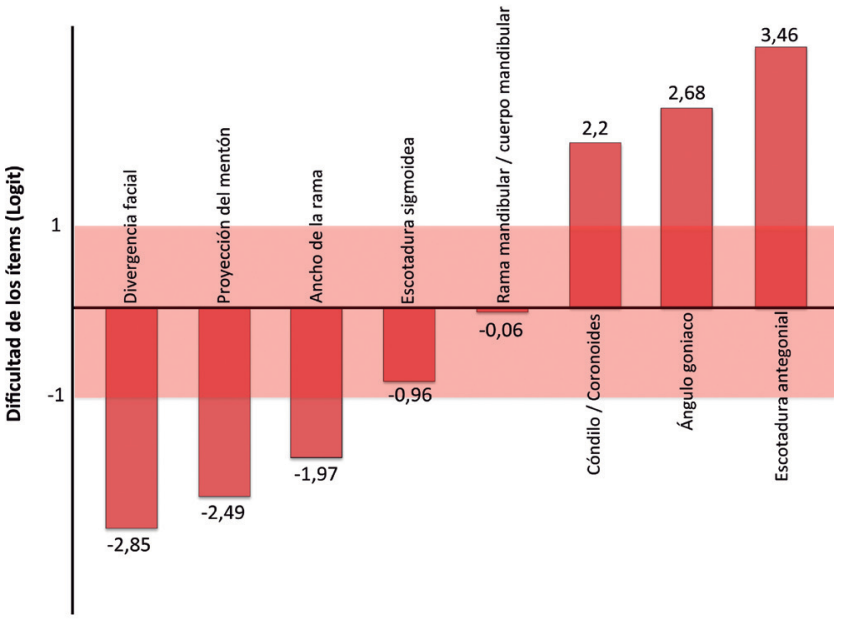

Gráfico 1. Grado de dificultad de los ítems

\section{DISCUSIÓN}

Existen varios análisis cefalométricos para determinar el biotipo facial, pero requieren de gran entrenamiento por parte del clínico. Ricketts en la década de 1960 desarrolló el VERT orientado al tratamiento ortodóncico de niños en crecimiento. Los análisis de Downs, Steiner y Jarabak no utilizaron explícitamente el concepto de biotipo facial, puesto que hasta ese momento no había sido definido.

En este estudio los ítems Proyección del mentón, Divergencia facial y Ancho de la rama mandibular obtuvieron un puntaje (Logit) menor a -1 , por lo que fueron clasificados como fáciles o muy fáciles. De ello se desprende que los estudiantes de 5to año son capaces de visualizar sin problemas esas estructuras en la telerradiografía con solo 10 minutos de entrenamiento, por lo que se podría construir una escala basada en estos parámetros.

Por otro lado, los ítems Relación de altura del cóndilo y coronoides, Ángulo goniaco y Escotadura antegonial, obtuvieron un puntaje (Logit) mayor a -1, por lo que se clasificaron con dificultad difícil o muy difícil. Si bien los ortodoncistas y cirujanos podrían identificar fácilmente todas las características, las más difíciles no deberían ser tomadas en cuenta para desarrollar una escala de identificación cualitativa del biotipo facial orientada a odontólogos generales.

\section{CONCLUSIONES}

Se determinó que los parámetros Relación entre la rama y el cuerpo mandibular, Proyección del mentón, Divergencia facial, Ancho de la rama mandibular y Escotadura sigmoidea son fáciles de visualizar en la telerradiografía de perfil, y por tanto útiles para evaluar el biotipo facial de manera sencilla y rápida.

\section{RESPONSABILIDADES ÉTICAS}

\section{Protección de personas y animales.}

Los autores declaran que para esta investigación no se han realizado experimentos en seres humanos ni en animales.

\section{Confidencialidad de los datos.}

Los autores declaran que han seguido los protocolos de su centro de trabajo sobre la publicación de datos de pacientes.

\section{Derecho a la privacidad y consentimiento informado.}

Los autores han obtenido el consentimiento informado de los pacientes y/o sujetos referidos en el artículo. Este documento obra en poder del autor de correspondencia.

\section{CONFLICTOS DE INTERÉS}

Los autores del trabajo declaran no tener conflicto de intereses.

\section{Bibliografía}

1. Gregoret J, Tuber E, Escobar L, Matos da Fonseca A. Ortodoncia y cirugía ortognática: Diagnóstico y planificación. Espaxs : Barcelona; 1997.

2. Bishara D, Jakobsen J. Longitudinal changes in three normal facial types. Am J Orthod. 1985;88(6):466-502

3. Le Pera F. Enfoque Nous-Biomecánico en el tratamiento del totalmente desdentado. Buenos Aires : Mundi; 1973

4. Mayoral J., Mayoral G. Ortodoncia. Principios fundamentales y prácticos. 6a. ed. Barcelona: Labor; 1990.

5. Farkas LG, Bryson W, Klotz J. Is photogrammetry of the face reliable? Plast Reconstr Surg. 1980;3(66):346-55

6 . Siriwat P, Jarabak J. Malocclusion and facial morphology; is there a relationship?.
Angle Orthod. 1985;55 (2):127-38.

7. Jarabak J, Fizzel J. Aparatología del arco de canto con alambres delgados. Técnica y tratamiento. Buenos Aires: Mundi; 1972.

8. Meyer J. Applied measurement with Jmetrik. New York: Routledge; 2014

9. Beszkin E, Lipszyc M, Voronovitsky L, Zielinsky L. Cefalometría clínica. 1a ed. Buenos Aires: Mundi; 1966.

10. Alarcón J.A., et al. Variation of mandibular sexual dimorphism across human facial patterns. Homo. 2016;67(3):188-202.

11. Prieto G, Delgado A. Análisis de un test mediante el Modelo Rasch. Psicothema. 2003;15(1):94-100. 